International Journal of Engineering \& Technology, $7(3.29)(2018) 243-248$
SPC
Website $: \frac{w w \text {. sciencepubco.com/index.php/IJET }}{\text { Research paper }}$

\title{
Assessment of different turbulence models in simulating axisymmetric flow in suddenly expanded nozzles
}

\author{
Sher Afghan Khan ${ }^{1}{ }^{*}$, Mir Owais Ali ${ }^{1}$, Miah Mohammed Riyadh ${ }^{1}$, Zahid Hossen ${ }^{1}$, Nafis Mahdi Arefin ${ }^{1}$ \\ ${ }^{1}$ Mechanical Engineering Dept., Faculty of Engineering, International Islamic University Malaysia, Kuala Lumpur \\ *Corresponding author E-mail: sakhan@iium.edu.my
}

\begin{abstract}
A numerical simulation was carried out to compare various turbulence models simulating axisymmetric nozzle flow past suddenly expanded ducts. The simulations were done for $\mathrm{L} / \mathrm{D}=10$. The convergent-divergent nozzle has been modeled and simulated using the turbulence models: The Standard k- $\varepsilon$ model, The Standard k- $\omega$ model and The SST k- $\omega$ model. Numerical simulations were done for Mach numbers $1.87,2.2$, and 2.58 and the nozzles were operated for NPRs in the range from 3 to 11 . From the numerical analysis it is apparent that for a given Mach number and effect of NPR will result in maximum gain or loss of pressure. Numerical results are in good agreement with the experimental results.
\end{abstract}

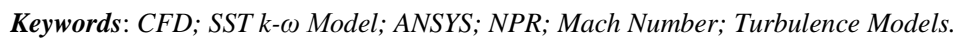

\section{Introduction}

The sudden expansion in both regimes of subsonic and supersonic flow is a prominent problem with a wide range of applications in Aerospace and Automobile industry. The abrupt expansion of flow in supersonic regimes is a very common problem arises in many industrial applications. Turbulent flow is considered by the irregular movement of elements of the fluid in terms of fluid dynamics. Turbulent flow is different than laminar flow because the fluid does not flow in similar layers. The cross mixing is considerably high in turbulent flow compared to the laminar flow. Turbulent flow can be categorized by recirculation, vortexes, and apparent randomness. The speed of the fluid in turbulent flow at a point goes through continuous changes in both magnitude and direction. One of the main tools available for their study is CFD analysis, if we do not have the proper experimental facility. Different turbulence models have been used for the simulation of the CD nozzle flow and each of these models have certain advantages as well as disadvantages over others. The $\mathrm{k}-\varepsilon$ model solves for two variables which are $\mathrm{k}$ and $\varepsilon$ (epsilon). In this model $\mathrm{k}$ is the turbulence kinetic energy and $\varepsilon$ (epsilon) is the rate of dissipation of turbulence kinetic energy. Wall functions are used in this model and because of that the flow in the buffer region is not simulated. The $\mathrm{k}-\omega$ model is almost identical as the $\mathrm{k}-\varepsilon$ model, but it explains for $\omega$ (omega), which is the specific rate of dissipation of kinetic energy. In combination with wall function it also can be used. Compared to the k- $\varepsilon$ model, the $\mathrm{k}-\omega$ model is more nonlinear, and that is why it is more difficult to converge. The SST model is a combination of the $\mathrm{k}-\varepsilon$ model in the free stream and the $\mathrm{k}-\omega$ model near the walls. It is a low Reynolds number model and kind of the go-to model for industrial purposes. It consists of almost identical resolution requirements to the $\mathrm{k}-\omega$ model and the low Reynolds number k- $\varepsilon$ model. The weaknesses displayed by standard k- $\omega$ and $k-\varepsilon$ models are eliminated by the SST model [1].

CFD ANSYS FLUID software has been used for the design procedure of the CD nozzle. It is a simulating software that is utilized for simulating computer models of structures, electronics, or machine components to simulate strength, toughness, elasticity, temperature distribution, electromagnetism, fluid flow, and other attributes modeling fluid flow and heat transfer in complex geometries. This simulation software has the capability of doing complete meshing flexibility as well as the ability to resolve flow problems utilizing structured or unstructured mesh that can be generated for complex geometries. Meshing in this software can include 2D/3D triangular or quadrilateral and many more. In the software "ANSYS" large structures can be broken down into smaller elements or divisions which can be solved as unique and individual components. Here the geometry of the object is created then dimensions are added, and then weight, pressure, temperature and other physical properties are added along with it [2].

The investigation outlined in this paper is the design of a supersonic convergent-divergent (CD) Nozzle on the assumption on the compressible flow of an Ideal gas. The effects of several NPRs (Nozzle Pressure Ratio) are considered for Mach numbers 1.87, 2.2, 2.58 . The pressure and the velocity in the expanded duct have been observed from the contours, figures, and plots.

\section{Literature review}

The data obtained from experimental studies carried out from various studies have evaluated the accuracy of the turbulence model for expecting the flow field and the nozzle performance precisely. Two-dimensional axisymmetric compressible flow analysis through a CD nozzle has been studied with the help of ANSYS FLUENT by using K- $\varepsilon$ turbulence model and Spalart-Allmaras turbulence model, a comparative investigation between the models on the basis pressure, velocity, temperature contours and vectors to ascertain the efficient design conditions for $\mathrm{CD}$ nozzles also been done [3].

The flow field of axisymmetric expansion shows a complex phenomena which are characterized by flow separation, reattachment, and recirculation. This kind of flow can be divided into two regions 
by a shear layer, one of them being the flow recirculation region and the other being the main flow region [4]. The realizable $k-\varepsilon$ model has been proposed from the comparison of the turbulence models [5]. Realizable k- $\varepsilon$ model gives quite good result for flat and round jets. It demonstrates the distribution of the dissipation rate. For large pressure ratio and separated and flows, it shows good assumption of the boundary layers [6]. In the year 1993 SST k- $\omega$ (Shear Stress Transport) turbulent model was introduced. $k-\omega$ and $\mathrm{k}-\varepsilon$ models are very similar. The $\mathrm{k}-\omega$ model uses equation for the turbulent energy instead of dissipation equation to find the dissipation rate $\omega . \mathrm{k}$ determines the energy of turbulence. $\omega$ determines the characteristic linear turbulence scale [7]. Flow near wall with the large pressure gradients is described using this model. However, for jet streams it faces problems in the calculation. The $\mathrm{k}-\omega$ model is sensitive to the initial conditions. The SST k- $\omega$ model (Shear Stress Transport) soon turned out be very commonly used. It was found out that the SST k- $\omega$ shows good results in mixing layers at medium pressure gradients. The SST k- $\omega$ model generates too high turbulence levels. In the calculated flow pattern, it leads to a massive change in quality. SST model lets us to define turbulence more precisely. Calculation of supersonic flows for advanced jet engines shows that the realizable k- $\varepsilon$ and transition SST turbulence models give the best results [6].

Turbulence models for turbulent flow over backward facing step shows turbulent flow is a very challenging task. The ability of different turbulence models to assume the reattachment distance in low Reynolds number is very different. " $\varepsilon$ " based "turbulence models" underpredict the reattachment length of flow. The recirculation created by backward facing step (BFS) is predictable by all turbulence models. The flow reattachment can be predicted well by SST models. The separation point is fixed from the assessment of 3D and 2D outcomes. Due to 3D nature of turbulent flow the reattachment points were different. The prediction of the reattachment point is the method to measure the precision of any numerical system. Velocity along the channel becomes positive for limitation in the distance from the step to the position. The primary method will give a rough estimation of reattachment length. Then the next method will give very accurate results [8].

\section{Methodology}

The process of a CFD based study follows the creation of geometry and mesh generation. Then we select the physics and fluid properties followed by the specification of boundary conditions. Initialization and solution control is done and the convergence is monitored. Post processing is done before the verification and validation of the results [9].

The geometries of the models shown in Figure 1 and Table 1 was taken from [10]. The specifications of the models which were used for the design Mach numbers are given in Table 1.

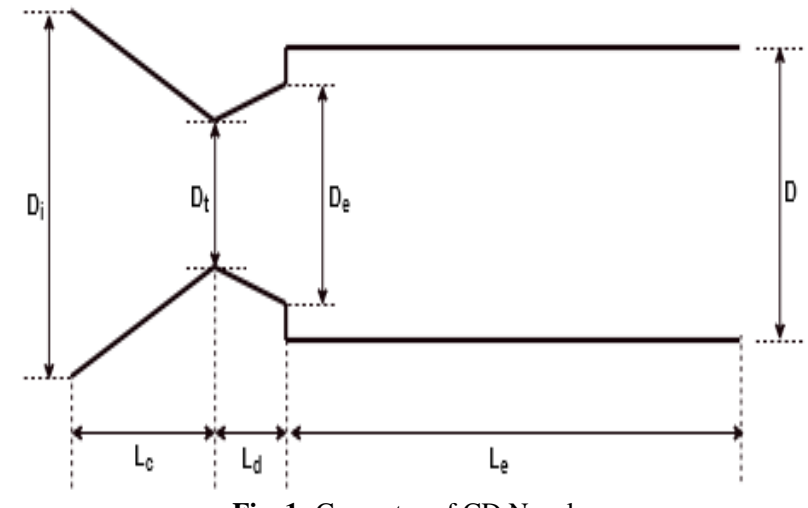

Fig. 1: Geometry of CD Nozzle.
Table 1: Dimensions of the CD Nozzle for Different Mach Numbers

\begin{tabular}{llll}
\hline & $\begin{array}{l}\text { Model 1 } \\
\text { (Mach-1.87) }\end{array}$ & $\begin{array}{l}\text { Model 2 } \\
\text { (Mach-2.2) }\end{array}$ & $\begin{array}{l}\text { Model 3 } \\
\text { (mach-2.58) }\end{array}$ \\
\hline $\begin{array}{l}\text { Inlet Diameter } \\
\text { (Di) }\end{array}$ & 28.72 & 26.523 & 25.677 \\
$\begin{array}{l}\text { Throat Diameter } \\
\text { (Dt) }\end{array}$ & 8.648 & 6.45 & 5.605 \\
$\begin{array}{l}\text { Exit Diameter } \\
\text { (de) }\end{array}$ & 10 & 10 & 10 \\
$\begin{array}{l}\text { Extended diam- } \\
\text { eter (D) }\end{array}$ & 16 & 16 & 16 \\
$\begin{array}{l}\text { Convergent } \\
\text { Length (Lc) }\end{array}$ & 35 & 35 & 35 \\
$\begin{array}{l}\text { Divergent } \\
\text { length (Ld) }\end{array}$ & 12.926 & 16.88 & 20.907 \\
$\begin{array}{l}\text { Extended length } \\
\text { (Le) }\end{array}$ & 160 & 160 & 160 \\
\hline
\end{tabular}

The nozzle geometry is done by 2D planar body in ANSYS Workbench from the dimensions given in Table 1. Figure 2 shows the 2D planar finite element model for a particular design Mach number used. Separate geometries were constructed for each of the design Mach numbers.

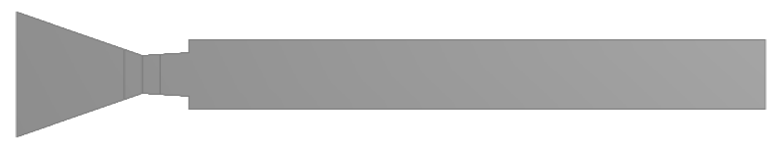

Fig. 2: 2D Model Made in ANSYS

The next procedure is to add mesh in the geometry. Meshing of a geometry is one of the most discussed issue in "CFD". The meshing determines the accuracy and stability of the simulation. The denser the meshing of the geometry, the higher the accuracy and stability of computation. For a C-D nozzle the most crucial parts are the walls of the nozzle and the throat and from Figure 3 one can observe that the density of the mesh was made higher near the walls and throat of the Nozzle.

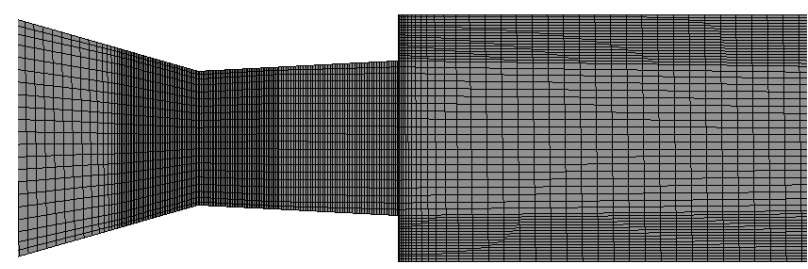

Fig. 3: Meshing of the Nozzle.

For the boundary condition specifications, the inlet was specified as pressure inlet, the outlet was specified as pressure outlet, the wall was assigned as wall. Pressure inlet boundary condition was used to define the fluid pressure at flow inlets. The inlet pressure for every case is calculated according to the NPR. The outlet pressure for every case is considered as 1 atmospheric pressure. 3, 5, 7, 9, 11 are the Nozzle Pressure Ratios used for the analysis. Wall boundary condition was used as stationary wall and no slip condition. For this study, the Standard k- $\varepsilon$ model, Standard k- $\omega$ model and SST k- $\omega$ model is used with energy equation on.

\section{Results and discussion}

The data displayed in this section consists of a comparison between the three simulated turbulence models (k- $\varepsilon, \mathrm{k}-\omega$, SST k- $\omega)$ and actual data measured by Baig et. al [10] for the wall pressure vs. the axial position measured after the exit of convergent-divergent nozzle. The data is compared for Mach numbers 1.87, 2.2 and 2.58 with each Mach number having Nozzle Pressure Ratios of 3,5,7,9, and 11. The data is then separated into 15 different curves for each nozzle pressure ratios. 


\section{Mesh convergence}

For all the three models the equations were converged before the simulation was conducted. The convergence for $\mathrm{k}-\varepsilon, \mathrm{k}-\omega$ and SST $\mathrm{k}-\omega$ model is as shown in figures $4-\mathrm{a}, 4-\mathrm{b}$, and 4-c respectively

(A)

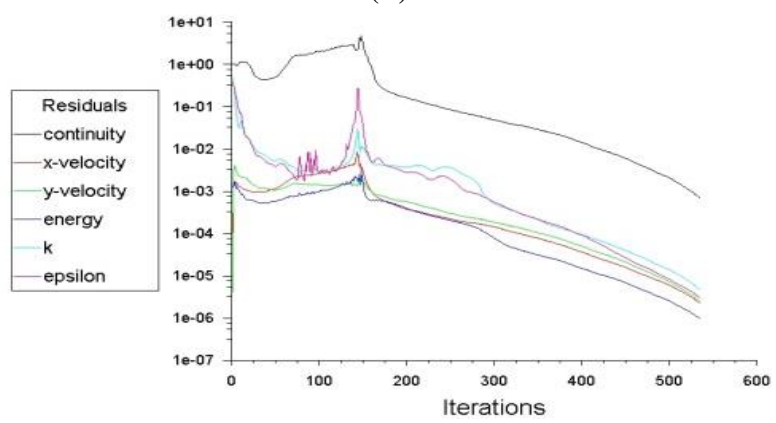

(B)

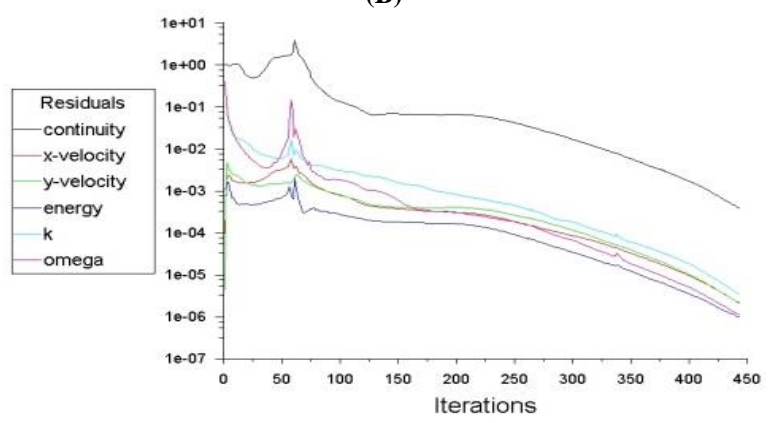

(C)

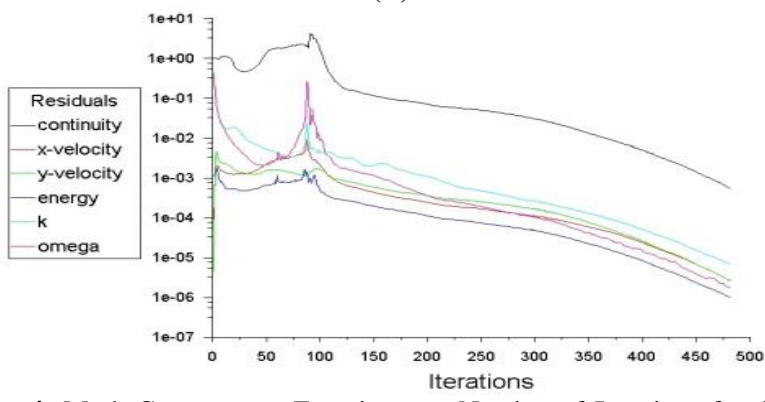

Fig. 4: Mesh Convergence Equations vs. Number of Iterations for three Models.

\section{Wall pressure data}

The measured data for wall pressure for all the Mach numbers is taken from [10]. The figure 5 shows the wall pressure vs. axial position (non-dimensional) for a Mach number of 1.87. The wall pressure is measured only after the flow is suddenly expanded and is exiting the convergent-divergent nozzle. Figure 5-a represents the results for NPR 3. Results acquired from the simulation for all three turbulence models show similar trends when compared with the actual values. Out of the three, the k- $\omega$ turbulence model matches closest. For this NPR, no shock is present when the nozzle suddenly expands and the flow is over-expanded when it leaves the convergent-divergent nozzle. Figure 5-b displays the results for NPR $=5$ of the same Mach No. The curves obtained from all turbulence models and the measured values are closely related. After flow reattachment, the values are almost identical. There is a presence of oblique shock waves and the flow is not completely over-expanded. For NPR $=7$ as shown in figure 5(c), the curves also show similar pattern and seem to converge with the actual values towards the nozzle exit. The $\mathrm{k}-\omega$ turbulence model produces the best result. The data for NPR $=9$ and NPR $=11$ are shown in figure 5(d) and 5(e) respectively and it can be observed that the curves are slightly separated of the three, the $\mathrm{k}-\omega$ model is seems to be the best fit. The flows for the last two NPRs are under-expanded upon exiting from the convergent-divergent nozzle.

(A)

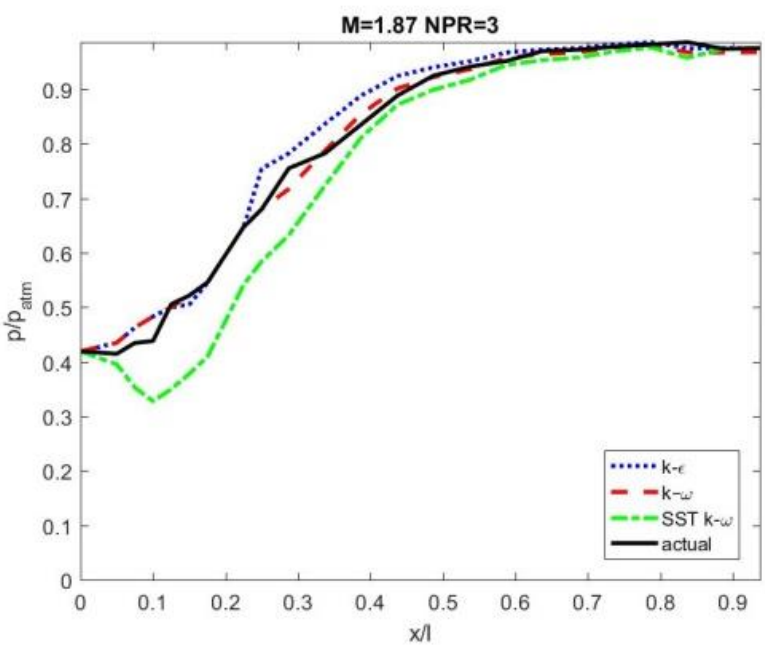

(B)

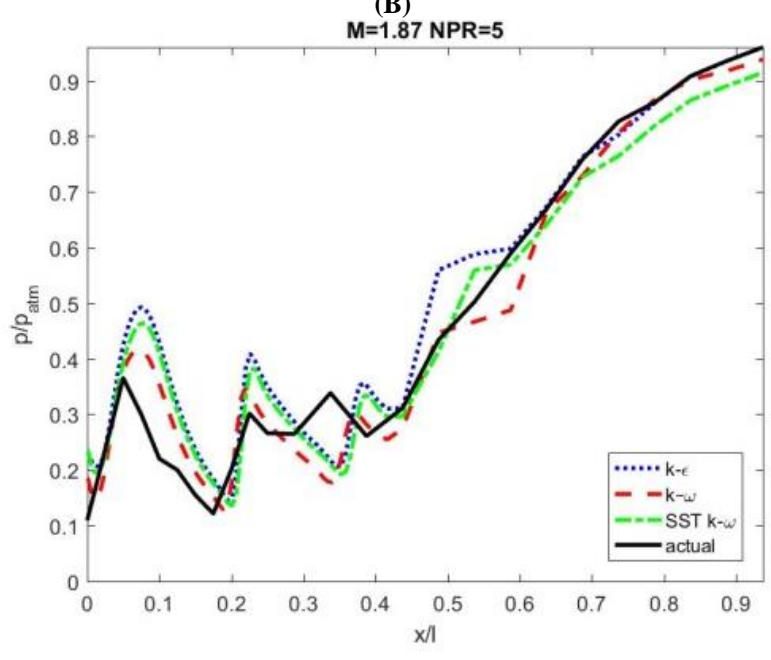

(C)

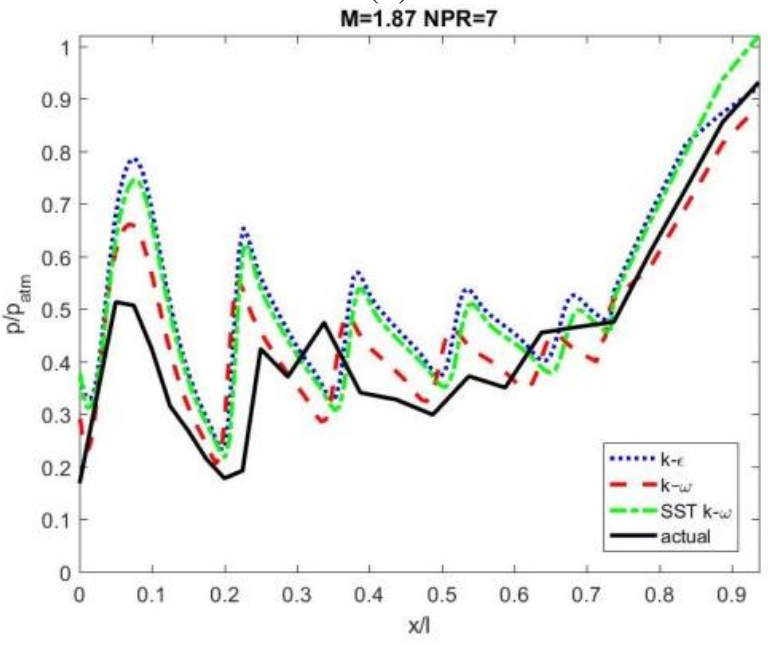


(D)

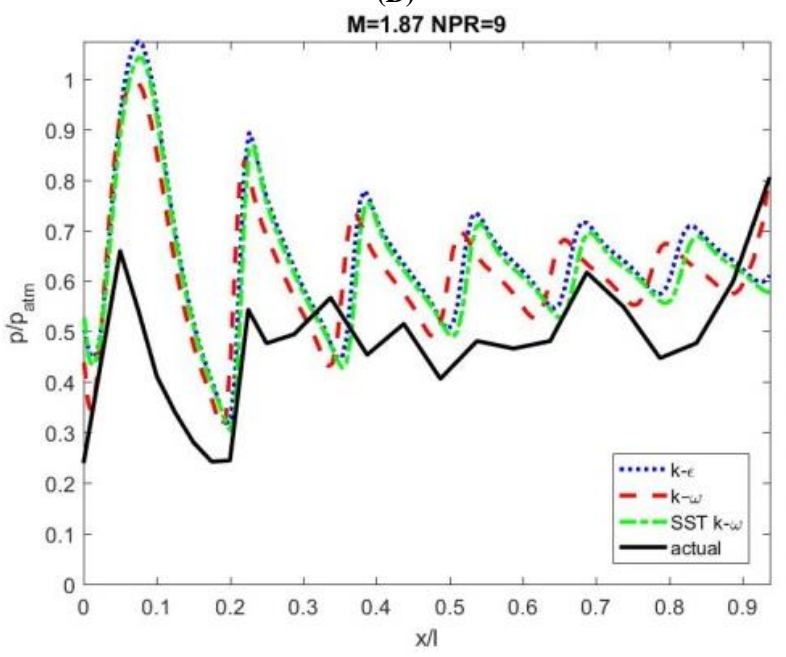

(E)

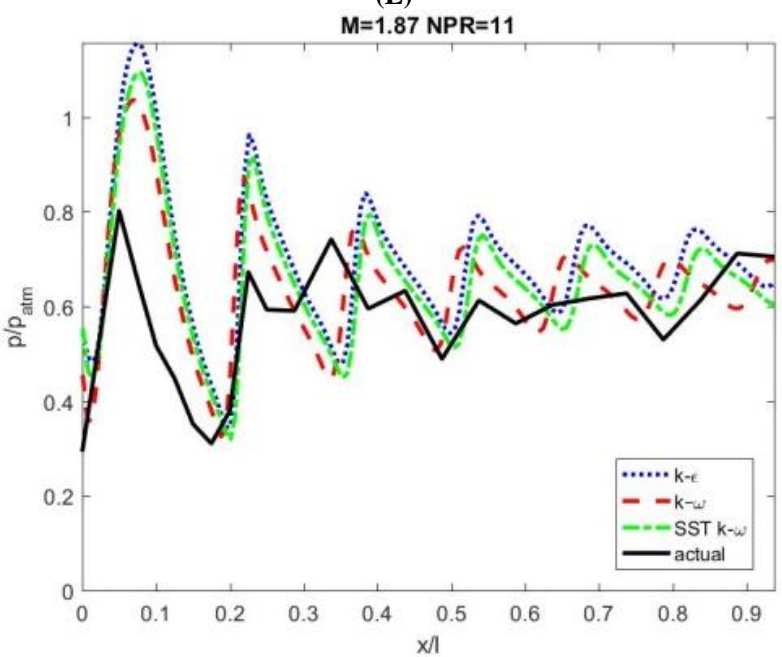

Fig. 5: Non-Dimensional Wall Pressure vs Non-Dimensional Axial Position for Mach number $=1.87$.

Now figure 6 shows the wall pressure distribution for Mach No. 2.2. The figures 6(a) and 6(b) represent NPR 3 and 5 respectively. The curves show very close characteristics when compared with the experimental values. In the subsequent figures $6(\mathrm{c}), 6(\mathrm{~d})$ and $6(\mathrm{e})$, are the curves for NPR 7, 9 and 11 respectively. They also show a similar pattern but the matching with experimental results is poor. Since at these NPRs the oblique shock waves are found. Here also, the best result is obtained from the k- $\omega$ turbulence model. The flow is over-expanded while exiting the nozzle for all the NPRs of the present study.

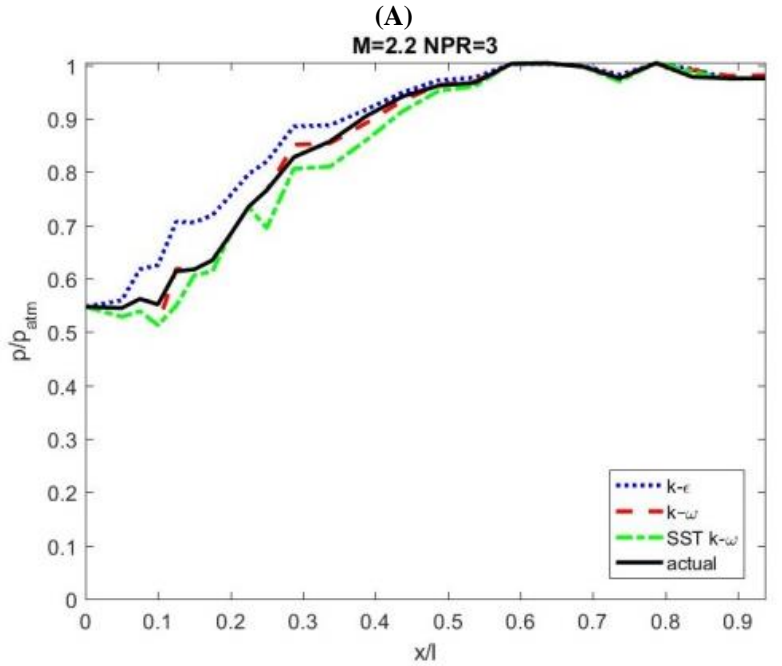

(B)

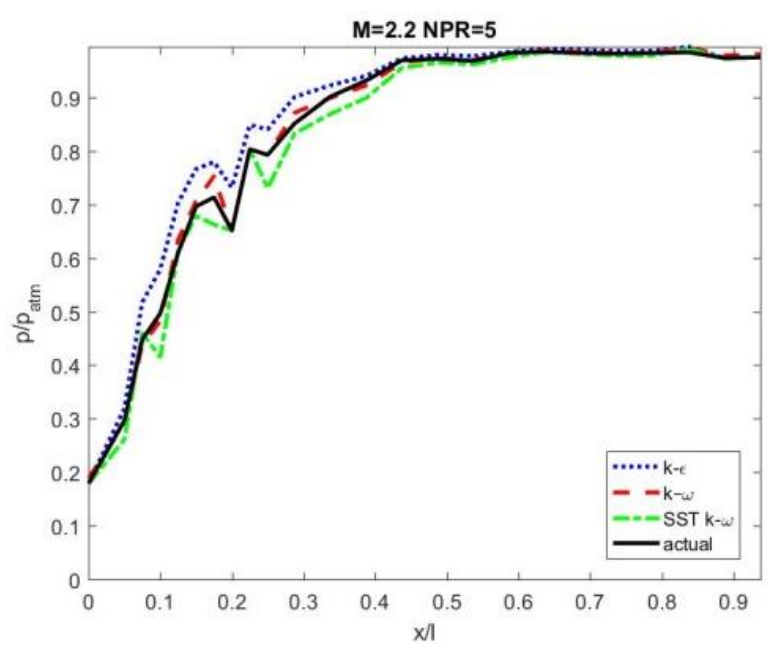

(C)

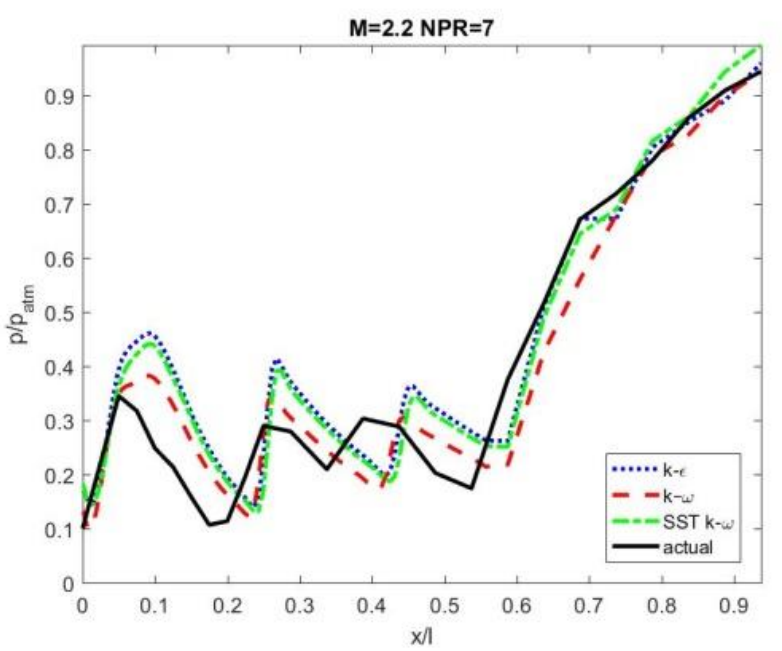

(D)

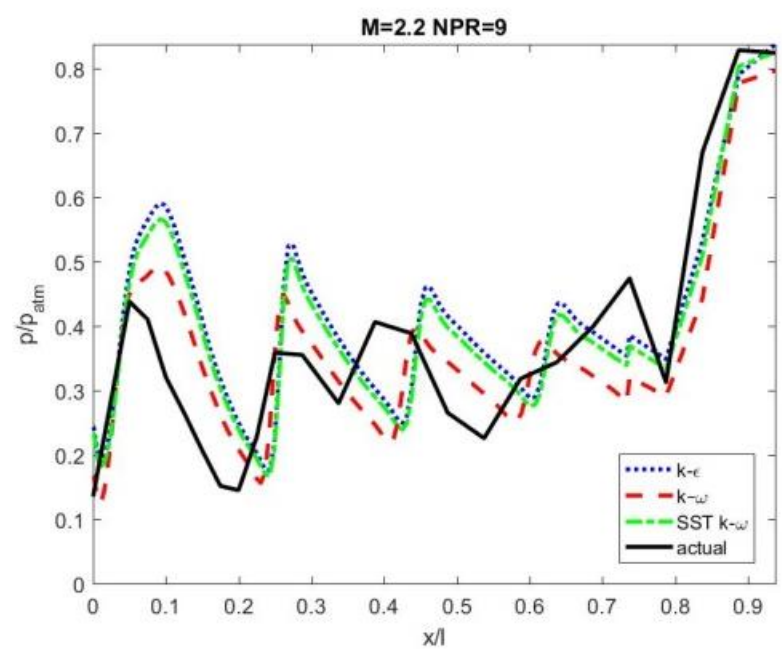


(E)

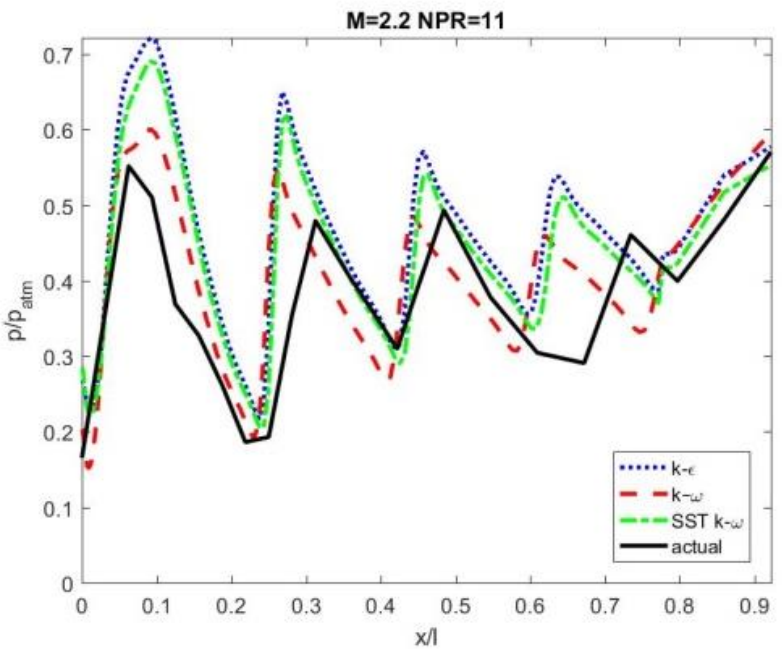

Fig. 7: Non-Dimensional Wall Pressure vs Non-Dimensional Axial Position for Mach number $=2.2$.

For Mach No. 2.58, wall pressures are shown in figures 7. From the curves of NPR 3, 5 and 7 as indicated in figures 7(a), 7(b) and 7(c) respectively, it can be observed that the plots are very close to the wind tunnel values and also jets remain over-expanded. The simulations results for NPR 9 and 11, are shown in figures 7(d) and 7(e), oblique shock waves are sitting at the exit but it diminishes after exiting the nozzle exit. The k- $\omega$ turbulence model has the closest values for this case as well.

(A)

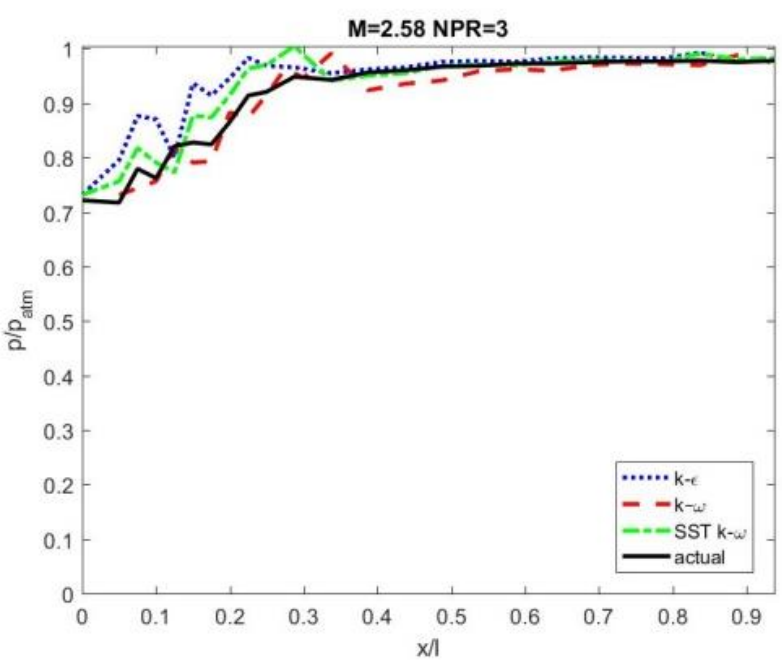

(B)

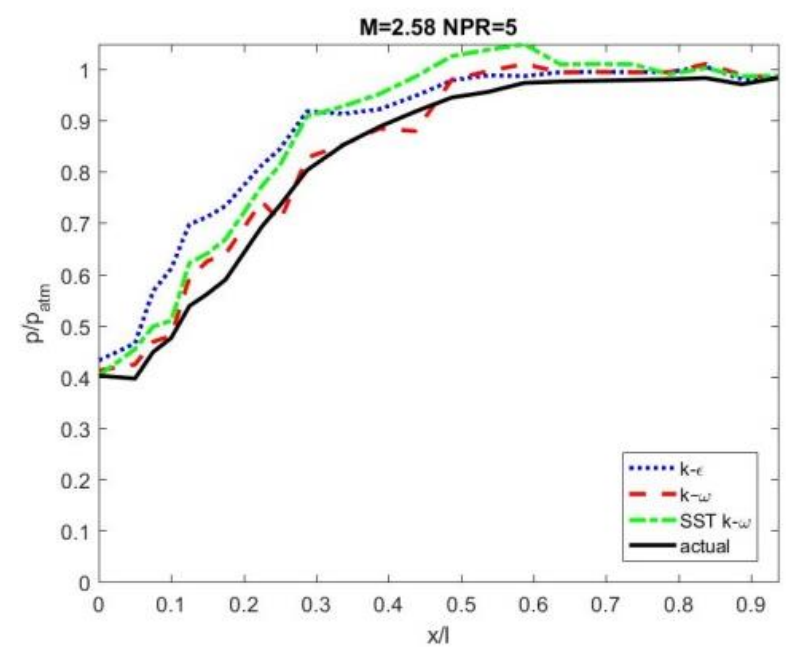

(C)

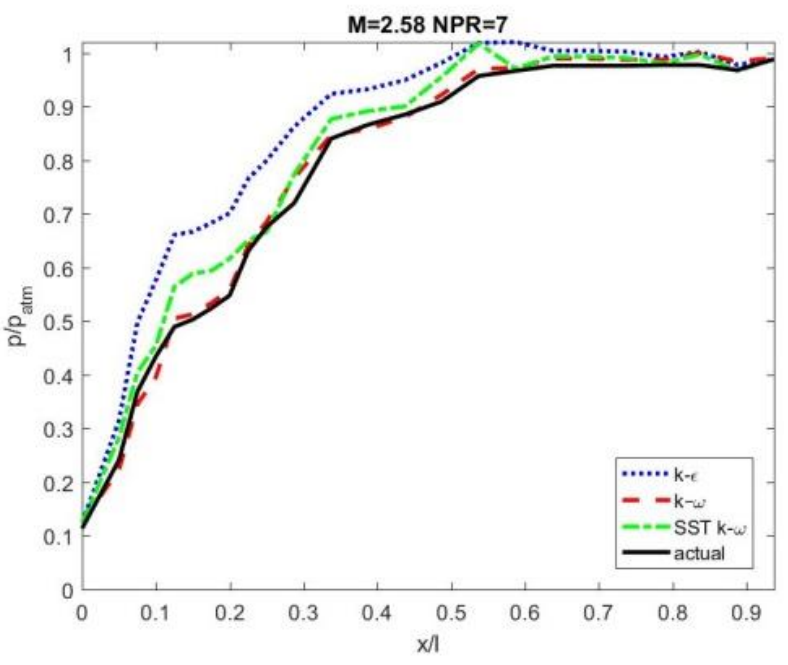

(D)

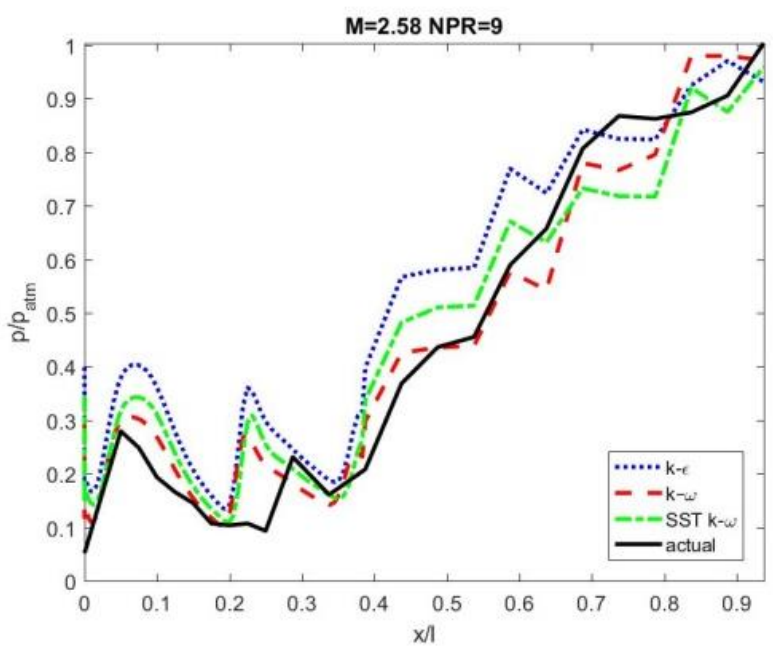

(E)

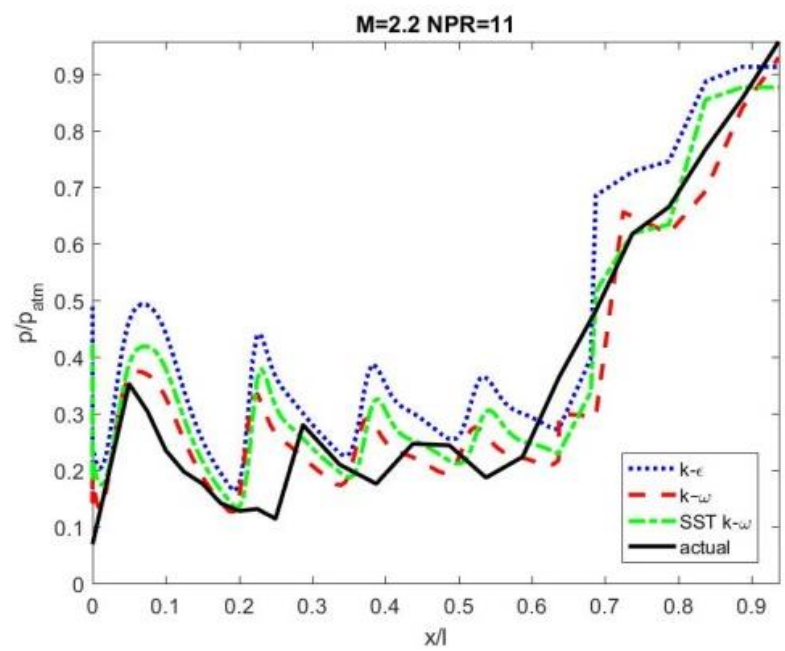

Fig. 7: Non-Dimensional Wall Pressure vs Non-Dimensional Axial Position for Mach number $=2.58$.

\section{Conclusion}

From the results obtained, the following conclusions can be made. The $\mathrm{k}-\omega$ turbulence model provides the best fit/solutions for all the cases. Also the experimentally measured data is verified by CFD analysis and they are very closed. For future work, k- $\omega$ turbulence model may be selected to find the CFD analysis of controlled flow. The data for controlled flow which was also measured by Baig et 
al. [10] can be compared with $\mathrm{k}-\omega$ turbulence model. The simulation were conducted only for $\mathrm{L} / \mathrm{D}=10$. For other $\mathrm{L} / \mathrm{D}$ ratios, the $\mathrm{k}$ $\omega$ model may also be selected as means of validation. Since all the curves indicated were closed to the experimental values their behavior is on the similar lines further, it is proved that the measured data and the CFD results match very well.

\section{References}

[1] Frei, W. (2013). Which Turbulence Model Should I Choose for my CFD Application? Retrieved July 19, 2018, from http:// www. comsol. com/blogs /which-turbulence-model-should-choose-cfd-application/.

[2] J. Sodja, "Turbulence models in CFD," Univ. Ljubljana, no. March, pp. 1-18, 2007.

[3] S. A. Khan and E. Rathakrishnan, "Active control of suddenly expanded flows from underexpanded nozzles - Part II," Int. J. Turbo Jet Engines, vol. 22, no. 3, pp. 163-183, 2005.

[4] S. A. Khan and A. Aabid, "Cfd Analysis of Cd Nozzle and Effect of Nozzle Pressure Ratio,” Int. J. Mech. Prod. Eng. Res. Dev., vol. 8, no. June, pp. 1147-1158, 2018.

[5] N. A. Najar, D. Dandotiya, and F. A. Najar, "Comparative Analysis of K-Epsilon and Spalart-Allmaras Turbulence Models for Compressible Flow Through a Convergent-Divergent Nozzle,” Int. J. Eng Sci., vol. 2, no. 8, pp. 8-17, 2013.

[6] M. P. Bulat and P. V. Bulat, "Comparison of turbulence models in the calculation of supersonic separated flows," World Appl. Sci. J., vol. 27, no. 10, pp. 1263-1266, 2013.

[7] F. R. Menter, "Improved two-equation k-omega turbulence models for aerodynamic flows," NASA Tech. Memo., no. 103978, pp. 1-31, 1992.

[8] Anwar-ul-Haque, F. Ahmad, S. Yamada, and S. R. Chaudhry, "Assessment of Turbulence Models for Turbulent Flow over Backward Facing Step," World Congr. Eng. 2007 Vol II, vol. II, pp. 1-6, 2007.

[9] Tu, J., Yeoh, G. H., \& Liu, C. (2013). Computational fluid dynamics: a practical approach Elsevier/Butterworth Heinemann.

[10] M. A. A. Baig, S. A. Khan, C. Ahmed Saleel, and E. Rathakrishnan, "Control of base flows with micro jet for area ratio of 6.25," ARPN J. Eng. Appl. Sci., vol. 7, no. 8, pp. 992-1002, 2012. 